\title{
DYNAMICS OF FLEXIBLE SHELLS AND SHARKOVSKIY'S PERIODICITY
}

\author{
VADIM A. KRYSKO, JAN AWREJCEWICZ, NATALYA E. SAVELEVA, \\ AND ANTON V. KRYSKO
}

Received 15 December 2005; Revised 6 June 2006; Accepted 14 June 2006

Complex vibration of flexible elastic shells subjected to transversal and sign-changeable local load in the frame of nonlinear classical theory is studied. A transition from partial to ordinary differential equations is carried out using the higher-order BubnovGalerkin approach. Numerical analysis is performed applying theoretical background of nonlinear dynamics and qualitative theory of differential equations. Mainly the so-called Sharkovskiy periodicity is studied.

Copyright (C) 2006 Vadim A. Krysko et al. This is an open access article distributed under the Creative Commons Attribution License, which permits unrestricted use, distribution, and reproduction in any medium, provided the original work is properly cited.

\section{Fundamental relations of flexible shells theory}

We investigate a shallow shell which occupied three-dimensional subspace of a space $R^{3}$ using a curvilinear system of coordinates $x, y, z$ introduced in the following way. In the shell body the middle surface is fixed for $z=0$; axes $o x$ and $o y$ are directed along the main curvatures of this surface, whereas the $o z$ axis is oriented into the curvature centre (Figure 1.1). In the given coordinates the shell is treated as the three-dimensional object $\Omega$ defined as follows:

$$
\Omega=\{x, y, z /(x, y, z) \in[0, \alpha] \times[0, \beta] \times[-h / 2, h / 2]\}
$$

We assume that the Lamè coefficients, $A, B$, and radii of the middle surface $R_{1}, R_{12}$, and $R_{2}$ are continuous functions of $x, y$ and their first derivatives. In contrary to the main radii $R_{1}, R_{2}$, the radii directed in $x, y$ are denoted by primes. Normal coordinate to the middle surface is denoted by $z$. Positive orientation of coordinates is shown in Figure 1.1. It is assumed that $h(x, y)$ does not have first-order discontinuities, and the maximal thickness $h_{\max } \equiv h_{0}$ is sufficiently smaller than a smallest main curvature radius $R_{\min }$. We assume that the quantity $h_{0} / R_{\min }$ can be neglected in comparison to 1 . Shells satisfying this assumption are called thin shells. We assume that the straight normal hypothesis holds 


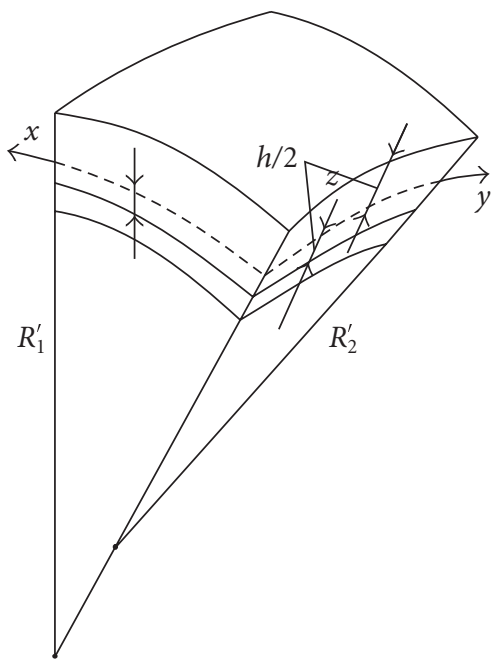

Figure 1.1

(Kirchhoff-Love hypothesis). We use the following differential equations and deformation continuity:

$$
\begin{gathered}
{\left[\frac{1}{\lambda^{2}} \frac{\partial^{2} w}{\partial x^{2}} \frac{\partial^{2}(\cdot)}{\partial x^{2}}+\lambda^{2} \frac{\partial^{2} w}{\partial y^{2}} \frac{\partial^{2}(\cdot)}{\partial y^{2}}+2(1-\mu) \frac{\partial^{2} w}{\partial x \partial y} \frac{\partial^{2}(\cdot)}{\partial x \partial y}+\mu\left(\frac{\partial^{2} w}{\partial x^{2}} \frac{\partial^{2}(\cdot)}{\partial y^{2}}+\frac{\partial^{2} w}{\partial y^{2}} \frac{\partial^{2}(\cdot)}{\partial x^{2}}\right)\right]} \\
-\nabla_{k}^{2} F+p_{x}(t) \frac{\partial^{2} w}{\partial y^{2}}+p_{y}(t) \frac{\partial^{2} w}{\partial x^{2}}-L(w, F)+M \cdot q(t)-\left(\frac{\partial^{2} w}{\partial t^{2}}+\varepsilon \frac{\partial w}{\partial t}\right)=0, \\
{\left[\left(\lambda^{2} \frac{\partial^{2} F}{\partial y^{2}}-\mu \frac{\partial^{2} F}{\partial x^{2}}\right) \frac{\partial^{2}(\cdot)}{\partial y^{2}}+\left(\frac{1}{\lambda^{2}} \frac{\partial^{2} F}{\partial x^{2}}-\mu \frac{\partial^{2} F}{\partial y^{2}}\right) \frac{\partial^{2}(\cdot)}{\partial x^{2}}+2(1+\mu) \frac{\partial^{2} F}{\partial x \partial y} \frac{\partial^{2}(\cdot)}{\partial x \partial y}\right]} \\
+\nabla_{k}^{2} w+\frac{1}{2} L(w, w)=0,
\end{gathered}
$$

where

$$
L(w, F)=\frac{\partial^{2} w}{\partial x^{2}} \frac{\partial^{2} F}{\partial y^{2}}+\frac{\partial^{2} F}{\partial x^{2}} \frac{\partial^{2} w}{\partial y^{2}}-2 \frac{\partial^{2} w}{\partial x \partial y} \frac{\partial^{2} F}{\partial x \partial y}, \quad \nabla_{k}^{2}=k_{y} \frac{\partial^{2}}{\partial x^{2}}+k_{x} \frac{\partial^{2}}{\partial y^{2}} .
$$

System of (1.2) is reduced to the nondimensional form by introduction of the nondimensional quantities: $w=h \bar{w}, F=E h^{2} \bar{F}, t=t_{0} \bar{t}, \varepsilon=\bar{\varepsilon} / \tau$. Furthermore, for a spherical rectangular and cylindrical panels we have

$$
\begin{gathered}
x=a \bar{x}, \quad y=a \bar{y}, \quad k_{x}=\bar{k}_{x} \frac{h}{b^{2}}, \quad k_{y}=\bar{k}_{y} \frac{h}{a^{2}}, \quad q=\bar{q} \frac{E h^{4}}{a^{2} b^{2}}, \\
p_{x}=\bar{p}_{x} \frac{E h^{3}}{b^{2}}, \quad p_{y}=\bar{p}_{y} \frac{E h^{3}}{a^{2}}, \quad \tau=\frac{a b}{h} \sqrt{\frac{\rho}{E g}}, \quad M=1, \quad \lambda=\frac{a}{b},
\end{gathered}
$$


where $a$ and $b$ are shell dimensions associated with $x$ and $y$, respectively. For a closed cylindrical shell we have

$$
\begin{gathered}
x=L \bar{x}, \quad y=R \bar{y}, \quad k_{y}=\bar{k}_{y} \frac{h}{R^{2}} \quad\left(k_{x}=0\right), \quad q=\bar{q} \frac{E h^{4}}{L^{2} R^{2}}, \\
p_{x}=\bar{p}_{x} \frac{E h^{3}}{R^{2}}, \quad p_{y}=\bar{p}_{y} \frac{E h^{3}}{L^{2}}, \quad \tau=\frac{L R}{h} \sqrt{\frac{\rho}{E g}}, \quad M=k_{y}^{2}, \quad \lambda=\frac{L}{R}, \quad
\end{gathered}
$$

where $L$ and $R=R_{y}$ denote length and radius of the shell, respectively. In addition $t$ denotes time, $\varepsilon$ denotes the damping coefficient of a shell surrounding medium, $\mu=0.3$, and $p_{x}(t), p_{y}(t)$ are longitudinal loads, whereas $q(x, y, t)$ is the transversal load. Now and further bars standing over nondimensional quantities are omitted.

The following boundary conditions are added to (1.2):

$$
\begin{gathered}
w=0, \quad \frac{\partial^{2} w}{\partial x^{2}}=0, \quad F=0, \quad \frac{\partial^{2} F}{\partial x^{2}}=0 \quad \text { for } x=0 ; 1, \\
w=g(x, y, t), \quad \frac{\partial^{2} w}{\partial y^{2}}=r(x, y, t), \quad F=u(x, y, t), \quad \frac{\partial^{2} F}{\partial y^{2}}=z(x, y, t) \quad \text { for } y=0 ; \xi,
\end{gathered}
$$

which describe ball-type support on the shell-end face with occurrence of flexible ribs. We take $\xi=2 \pi$ for a closed cylindrical shell, and $\xi=1$ for both cylindrical and spherical panels. Note that an occurrence of nonhomogeneities of the boundary conditions occurred in second rows (for $y=0 ; \xi$ ) exhibits existence of initial imperfections and stresses in the shell. The following initial conditions are attached:

$$
\left.w\right|_{t=0}=w_{0},\left.\quad \dot{w}\right|_{t=0}=0 .
$$

\section{The Bubnov-Galerkin algorithm}

In order to solve equations the functions $w$ and $F$, being their solutions, are approximated by an analytical solution containing a finite number of arbitrary parameters in the following form:

$$
w=\sum_{i=0}^{N_{1}} \sum_{j=0}^{N_{2}} A_{i j}(t) \varphi_{i j}(x, y), \quad F=\sum_{i=0}^{N_{1}} \sum_{j=0}^{N_{2}} B_{i j}(t) \psi_{i j}(x, y),
$$

and the initial conditions (1.7) are applied.

In order to find an approximate value of elements $w$ and $F$, the following coordinate function systems are taken: $\left\{\varphi_{i j}(x, y), \psi_{i j}(x, y)\right\}(i, j=0,1,2, \ldots)$ in $(2.1)$, which satisfy the following requirements:

(1) $\varphi_{i j}(x, y) \in H_{A}, \psi_{i j}(x, y) \in H_{A}$, where $H_{A}$ is a Hilbert space, which is also referred as an energetic space;

(2) for all $i$ and $j$ both functions $\varphi_{i j}(x, y)$ and $\psi_{i j}(x, y)$ are linearly independent, continuous together with their partial derivatives to the fourth order in $\Omega$; 
4 Dynamics of flexible shells and Sharkovskiy's periodicity

(3) $\varphi_{i j}(x, y)$ and $\psi_{i j}(x, y)$ satisfy the main boundary conditions (and initial conditions) exactly;

(4) $\varphi_{i j}(x, y)$ and $\psi_{i j}(x, y)$ are complete in $H_{A}$;

(5) $\varphi_{i j}(x, y)$ and $\psi_{i j}(x, y)$ should represent $N$ first elements of the complete functions system.

Coefficients $A_{i j}(t)$ and $B_{i j}(t)$ are being the sought functions. Let us denote left-hand sides of system (1.2) by $\phi_{1}$ and $\phi_{2}$, respectively,

$$
\phi_{1}\left(w, F, \frac{\partial^{2} w}{\partial x^{2}}, \frac{\partial^{2} F}{\partial x^{2}}, \ldots\right)+M \cdot q(x, y, t)=0, \quad \phi_{2}\left(w, F, \frac{\partial^{2} w}{\partial x^{2}}, \frac{\partial^{2} F}{\partial x^{2}}, \ldots\right)=0
$$

Applying the Bubnov-Galerkin procedure to (2.2) one gets

$$
\begin{aligned}
& \int_{0}^{1} \int_{0}^{\xi} \phi_{1} \varphi_{r s}(x, y) d x d y+\int_{x_{1}}^{x_{2}} \int_{y_{1}}^{y_{2}} M q(x, y, t) \varphi_{r s}(x, y) d x d y=0, \\
& \int_{0}^{1} \int_{0}^{\xi} \phi_{2} \psi_{r s}(x, y) d x d y=0, \quad r=0,1, \ldots, N_{1} ; s=0,1, \ldots, N_{2} .
\end{aligned}
$$

Taking into account (2.3) equations (2.2) read

$$
\begin{gathered}
\sum_{r s}\left[\sum_{i j} A_{i j} \sum_{k l} H_{i j k l r s}+\sum_{i j} B_{i j} C_{1, i j r s}+\sum_{i j} A_{i j} W_{i j r s}+M q Q_{r s}\right. \\
\left.+\sum_{i j} A_{i j} \sum_{k l} B_{k l} D_{1, i j k l r s}+\sum_{i j}\left[\frac{d^{2} A_{i j}}{d t^{2}}+\varepsilon \frac{d A_{i j}}{d t}\right] G_{i j r s}\right]=0, \\
\sum_{r s}\left[\sum_{i j} A_{i j} C_{2, i j r s}+\sum_{i j} B_{i j} \sum_{k l} P_{i j k l r s}+\sum_{i j} A_{i j} \sum_{k l} A_{r s} D_{2, i j k l r s}\right]=0 .
\end{gathered}
$$

The symbol $\sum_{r s}[*]$ standing before each equation of system (2.4) shows that this equation is understood as a system of $r s$ such equations, and the integrals of the BubnovGalerkin approach follow:

$$
\begin{gathered}
H_{i j k l r s}=\int_{0}^{1} \int_{0}^{\xi} \frac{1}{12\left(1-\mu^{2}\right)}\left[\frac{1}{\lambda^{2}} \frac{\partial^{2} \varphi_{i j}}{\partial x^{2}} \frac{\partial^{2} \varphi_{k l}}{\partial x^{2}}+\lambda^{2} \frac{\partial^{2} \varphi_{i j}}{\partial y^{2}} \frac{\partial^{2} \varphi_{k l}}{\partial y^{2}}+2 \frac{\partial^{2} \varphi_{i j}}{\partial x \partial y} \frac{\partial^{2} \varphi_{k l}}{\partial x \partial x}\right] \varphi_{r s} d x d y, \\
C_{1, i j r s}=\int_{0}^{1} \int_{0}^{\xi}\left[-k_{y} \frac{\partial^{2} \psi_{i j}}{\partial x^{2}}-k_{x} \frac{\partial^{2} \psi_{i j}}{\partial y^{2}}\right] \varphi_{r s} d x d y, \\
C_{2, i j r s}=\int_{0}^{1} \int_{0}^{\xi}\left[k_{y} \frac{\partial^{2} \varphi_{i j}}{\partial x^{2}}+k_{x} \frac{\partial^{2} \varphi_{i j}}{\partial y^{2}}\right] \psi_{r s} d x d y,
\end{gathered}
$$




$$
\begin{gathered}
D_{1, i j k l r s}=\int_{0}^{1} \int_{0}^{\xi}\left[-L\left(\varphi_{i j}, \psi_{k l}\right)\right] \varphi_{r s} d x d y, \quad D_{2, i j k l r s}=\int_{0}^{1} \int_{0}^{\xi} \frac{1}{2} L\left(\varphi_{i j}, \varphi_{k l}\right) \psi_{r s} d x d y, \\
P_{i j k l r s}=\int_{0}^{1} \int_{0}^{\xi}\left[\frac{1}{\lambda^{2}} \frac{\partial^{2} \psi_{i j}}{\partial x^{2}} \frac{\partial^{2} \psi_{k l}}{\partial x^{2}}+\lambda^{2} \frac{\partial^{2} \psi_{i j}}{\partial y^{2}} \frac{\partial^{2} \psi_{k l}}{\partial y^{2}}+2 \frac{\partial^{2} \psi_{i j}}{\partial x \partial y} \frac{\partial^{2} \psi k l}{\partial x \partial x}\right] \psi_{r s} d x d y, \\
G_{i j r s}=\int_{0}^{1} \int_{0}^{\xi}\left[-\varphi_{i j} \varphi_{r s}\right] d x d y, \quad Q_{r s}=\int_{x_{1}}^{x_{2}} \int_{y_{1}}^{y_{2}} M q(x, y, t) \varphi_{r s} d x d y, \\
W_{i j r s}=\int_{0}^{1} \int_{0}^{\xi}\left[p_{y}(x, t) \frac{\partial^{2} \varphi_{i j}}{\partial x^{2}}+p_{x}(y, t) \frac{\partial^{2} \varphi_{i j}}{\partial y^{2}}\right] \varphi_{r s} d x d y .
\end{gathered}
$$

Integrals (2) (excluding $Q_{r s}$, when a transversal load is not applied to the whole shell surface) are computed with respect to the whole middle shell surface. After application of the Bubnov-Galerkin method a system of ordinary differential equations with respect to the functions $A_{i j}(t)$ and $B_{i j}(t)$ is obtained, which possesses the following matrix form:

$$
\begin{gathered}
\mathbf{G}(\ddot{\mathbf{A}}+\varepsilon \dot{\mathbf{A}})+\mathbf{H A}+\mathbf{W A}+\mathbf{C}_{1} \mathbf{B}+\mathbf{D}_{1} \mathbf{A B}=\mathbf{Q} q(t), \\
\mathbf{C}_{2} \mathbf{A}+\mathbf{P B}+\mathbf{D}_{2} \mathbf{A A}=0,
\end{gathered}
$$

where $\mathbf{H}=\left\|H_{i j r s}\right\|, \mathbf{G}=\left\|G_{i j r s}\right\|, \mathbf{C}_{1}=\left\|C_{1 i j r s}\right\|, \mathbf{C}_{2}=\left\|C_{2 i j r s}\right\|, \mathbf{D}_{1}=\left\|D_{1 i j k l r s}\right\|, \mathbf{D}_{2}=$ $\left\|D_{2 i j k l r s}\right\|, \mathbf{W}=\left\|W_{i j r s}\right\|, \mathbf{P}=\left\|P_{i j r s}\right\|$-square matrices with dimensions $2 \cdot N_{1} \cdot N_{2} \times 2$. $N_{1} \cdot N_{2}, \mathbf{A}=\left\|A_{i j}\right\|, \mathbf{B}=\left\|B_{i j}\right\|, \mathbf{Q}=\left\|Q_{i j}\right\|$-matrices of dimensions $2 \cdot N_{1} \cdot N_{2} \times 1$.

Second equation of the system (2.6) is computed with respect to $\mathbf{B}$ by using the inverse matrix method on each time step as follows:

$$
\mathbf{B}=\left[-\mathbf{P}^{-1} \mathbf{D}_{2} \mathbf{A}-\mathbf{P}^{-1} \mathbf{C}_{2}\right] \mathbf{A} .
$$

Multiplying the first equation of system (2.6) by $\mathbf{G}^{-1}$ and denoting $\dot{\mathbf{A}}=\mathbf{R}$, one gets the following first-order differential equations:

$$
\begin{gathered}
\dot{\mathbf{A}}=\mathbf{R}, \\
\dot{\mathbf{R}}=-\bar{\varepsilon} \mathbf{R}-\left[\mathbf{G}^{-1} \mathbf{C}_{\mathbf{1}}+\mathbf{G}^{-1} \mathbf{D}_{\mathbf{1}} \mathbf{A}\right] \cdot \mathbf{B}-\mathbf{G}^{-1} \mathbf{H A}-\mathbf{G}^{-1} \mathbf{W A}+\mathbf{G}^{-1} \mathbf{Q} q(\bar{t}) .
\end{gathered}
$$

Observe that the mentioned operation is allowed, since the inversed matrices $\mathbf{G}^{-1}$ and $\mathbf{P}^{-1}$ exist, if the coordinate functions are linearly independent.

Boundary (1.6) and initial (1.7) conditions are added to (2.8), and the defined Cauchy problem is solved by the fourth order Runge-Kutta method. A computational time step is chosen using the Runge principle.

\section{Sharkovskiy periodicity exhibited by the obtained differential equations}

A fundamental question of turbulence follows: how to predict turbulence occurrence using stability and equilibrium concepts? Feigenbaum investigated intervals between successive bifurcations (period-doubling bifurcations) using a quadratic function, referred 
to as a logistic map of the form $y=x^{2}+c$. The obtained result by Feigenbaum is a universal one. Period-doubling scenario occurs not only for the iterations of $c x(1-x)$ but also in a wide class of mappings of an interval into itself like $x^{2}+c, c \cdot \sin (\pi x)$ and $c x^{2} \cdot \sin (\pi x)$, defined on the appropriate intervals.

The following question arises: are there any other periodic orbits? They should be repellors, since in a map only attractors can be shown. It occurs that an occurrence of an orbit of period 3 implies an occurrence of the orbits with the periods $n=1,2,3, \ldots$ A role of period 3 orbit has been studied by Li and Yorke [1]. However, their considerations belong to a particular case of the Sharkovskiy theorem published in [2].

Theorem 3.1 (Sharkovskiy [2]). Let I be a finite or infinite interval in R. Assume that the mapping $f: Z \rightarrow I$ is continuous. If there exists a point $f$ of period $n$, then there is also a point $f$ of period $k$ for every integer positive number $k, k>n$, belonging to the following Sharkovskiy series:

$$
\begin{aligned}
& 3, \quad 5, \quad 7, \quad 9, \ldots \\
& 2 \cdot 3, \quad 2 \cdot 5, \quad 2 \cdot 7, \quad 2 \cdot 9, \ldots \\
& 2^{2} \cdot 3,2^{2} \cdot 5,2^{2} \cdot 7, \quad 2^{2} \cdot 9, \ldots \\
& \ldots, \quad 2^{n}, \ldots, 2^{2}, 2^{1}, 1 .
\end{aligned}
$$

Remark 3.2. If there exists an orbit of an odd period larger than one, then the number of different periods is infinite.

The Sharkovskiy theorem is applicable only for real function given on a real interval. If, for instance, a function $f$ is defined by rotation of each circle point on the angle of $2 / n$, then orbits of all points have one and only one period $n$. In this case any other periods do not exist at all, and consequently, the Sharkovskiy theorem cannot be applied.

A construction of one-dimensional transformation for simple dynamical systems allows one to get qualitatively similar bifurcation mechanisms of transition of mechanical systems into chaos. In the case of a complex continual mechanical system, one may get a similar picture only in the case when it is approximated by one degree-of-freedom system. Although for continual systems, dynamics is of more complexity, but on the diagrams exhibiting vibration character versus the control parameters $\left\{q_{0}, \omega_{p}\right\}$ one may also find subspaces with periods $3 ; 2 \cdot 3 ; 5 ; 2 \cdot 5 ; 7 ; 9 ; 11 ; 13 ; \ldots$. Motivated by numerical computation we believe that the Sharkovskiy theorem can be extended to fit more complex class of functions governing nonlinear dynamics of shells.

Investigating vibrations of a cylindrical shell with $k_{y}=112.5$ and $\lambda=3$ subjected to transversal sign-changing load $q=q_{0} \sin \left(\omega_{p} t\right)$ applied to zone width $\varphi_{0}=6$, among chaotic zones, also periodic windows belonging to Sharkovskiy's series are found. Time histories (signals) in the shell center, phase portraits, power spectra, and Poincaré maps, as well as the modal characteristics $w\left(w_{y y}\right)$ for the orbits mentioned in the Sharkovskiy theorem are further reported. 
Table 3.1. Indicators of chaos.

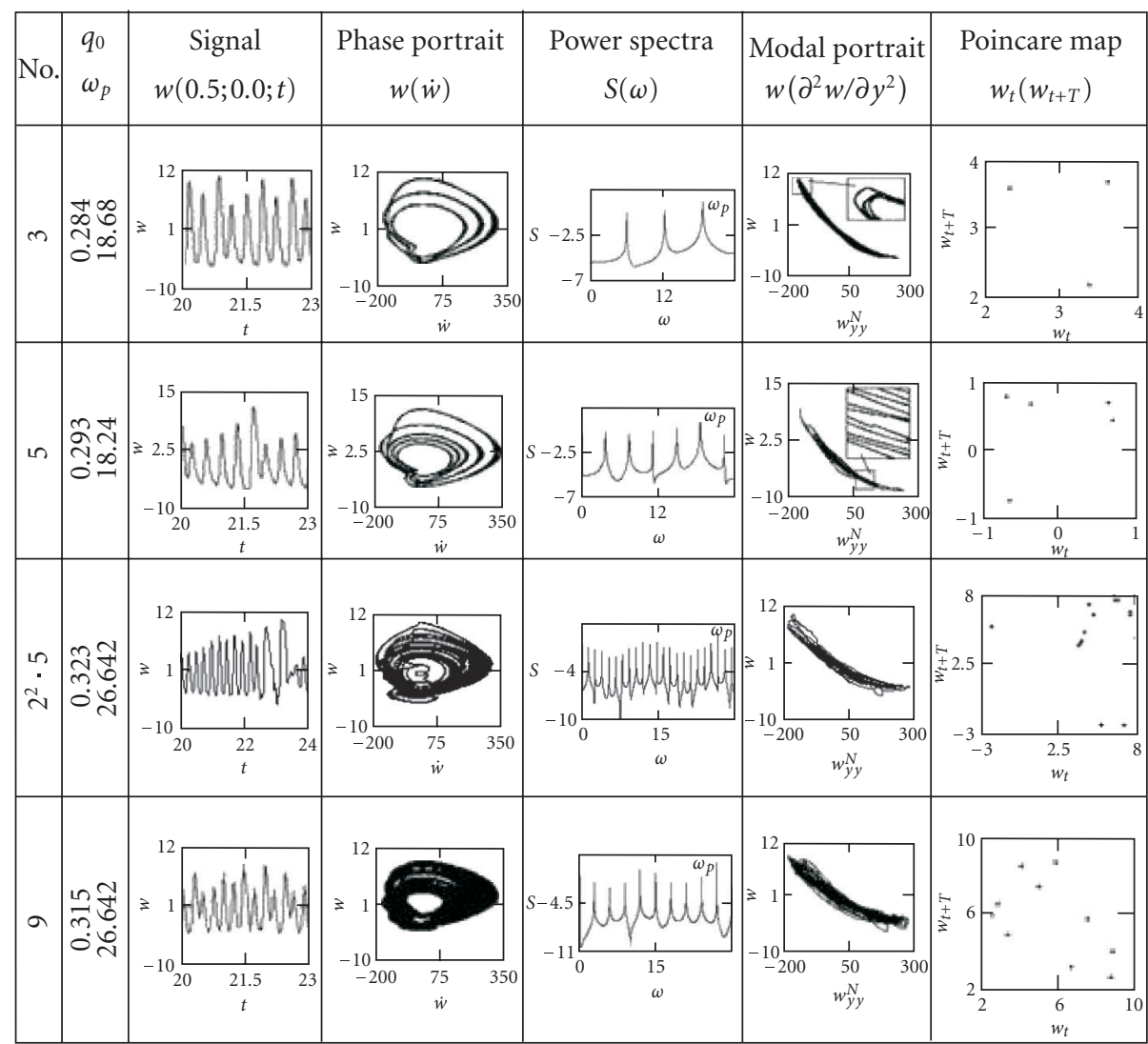

In Table 3.1 the following Sharkovskiy series is shown: $3 ; 5 ; 2^{2} \cdot 5 ; 9$. It should be emphasized that the so called Sharkovskiy series is not followed from each other, but they should be separated from the parameters $\left\{q_{0}, \omega_{0}\right\}$ space. The following scenarios are detected. In the case of period tripling a partition to three equal parts of the signal is observed; for period 5 we deal with 5 equal parts, and so on. In Poincaré maps one observes $3 ; 5 ; 2^{2} \cdot 5 ; 9$ points. In a phase portrait period doubling is observed too. The mentioned orbits are situated in the windows of regularity occurred in chaos and their structure is the same in the whole studied manifold.

Investigating complex vibrations of cylindrical panels subject to a longitudinal signchanging two parameters excitation of the form $p_{x}=p_{0} \sin \left(\omega_{p} t\right)$ and $p_{y}=\alpha \cdot p_{x}$ for $k_{y}=24$ and $\alpha=3$, besides chaotic zones, also periodic orbits being members of the Sharkovskiy series are detected. In Table 3.2 power spectra of the orbits mentioned in the Sharkovskiy theorem as well as the Sharkovskiy series $3 ; 2 \cdot 3 ; 5 ; 2 \cdot 5 ; 7 ; 2 \cdot 7 ; 11 ; 13$ are shown.

It should be emphasized that similar dynamical behavior has been found for both cylindrical panel as well as closed cylindrical shell. 
Table 3.2. Power spectra.

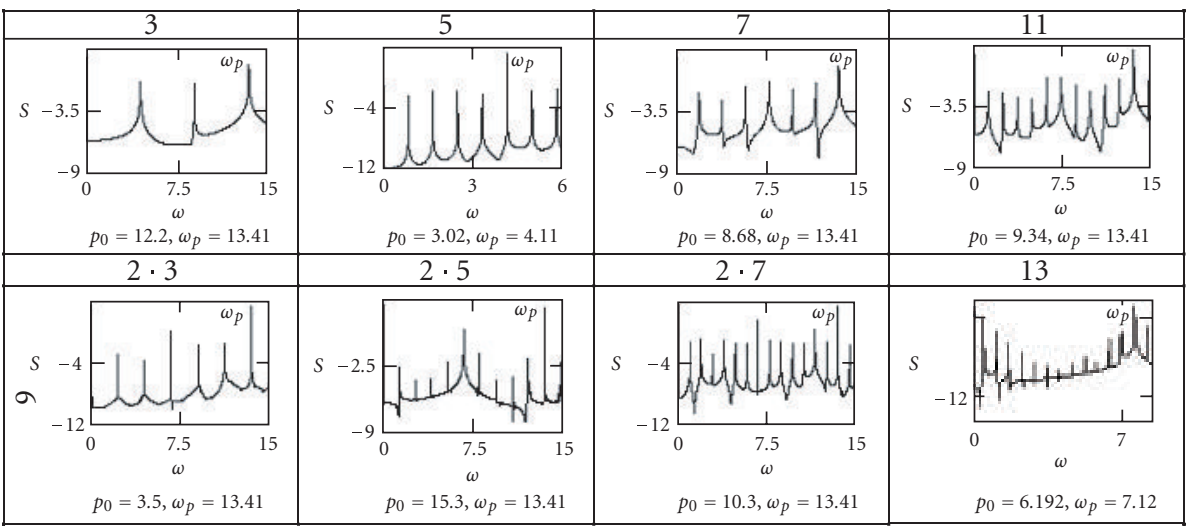

\section{Concluding remarks}

The Sharkovskiy periodicity for differential equations governing dynamics of flexible shells is reported. In the case of vibrations of the closed cylindrical shell subjected to uniformly distributed transversal load $q=q_{0} \sin \left(\omega_{p} t\right)$, in addition to the chaotic zones also the so-called Sharkovskiy series $3 ; 5 ; 2^{2} \cdot 5 ; 9$, have been detected. On the other hand, investigating the cylindrical panel subjected to longitudinal two-parameters load chaotic and periodic (being members of the Sharkovskiy series $3 ; 2 \cdot 3 ; 5 ; 2 \cdot 5 ; 7 ; 2 \cdot 7 ; 11 ; 13$ ) orbits have been detected.

\section{Acknowledgment}

The paper was presented at DSTA '05 (Dynamical Systems Theory and Applications) conference held in Łódź, December 12-15, 2005.

\section{References}

[1] T. Y. Li and J. A. Yorke, Period three implies chaos, The American Mathematical Monthly 82 (1975), no. 10, 985-992.

[2] A. N. Sharkovskiy, Existence of cycles of a continuous transformation of a straight line into itself, Ukrainian Mathematical Journal 26 (1964), no. 1, 6-71.

Vadim A. Krysko: Department of Mathematics, Saratov University, 410054 Saratov, Russia E-mail address: tak@san.ru

Jan Awrejcewicz: Department of Automatics and Biomechanics, Technical University of Łódź, 1/15 Stefanowskiego Street, 90-924 Łódź, Poland

E-mail address: awrejcew@p.lodz.pl

Natalya E. Saveleva: Department of Mathematics, Saratov University, 410054 Saratov, Russia E-mail address: qarx@mail.ru

Anton V. Krysko: Department of Mathematics, Saratov University, 410054 Saratov, Russia E-mail address: takk@san.ru 


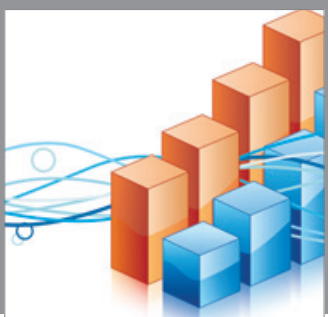

Advances in

Operations Research

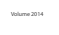

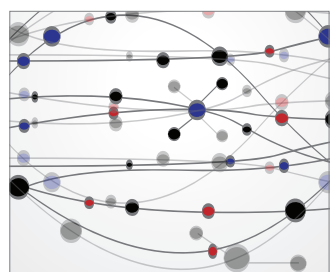

\section{The Scientific} World Journal
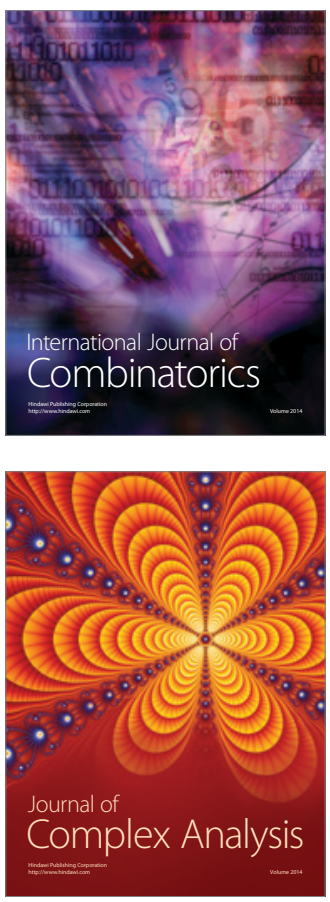

International Journal of

Mathematics and

Mathematical

Sciences
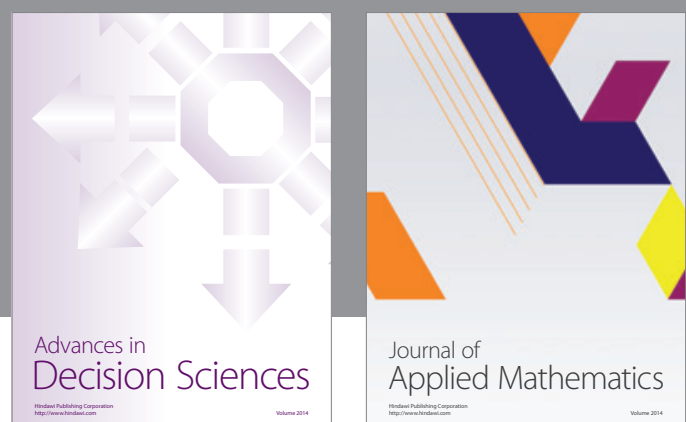

Journal of

Applied Mathematics
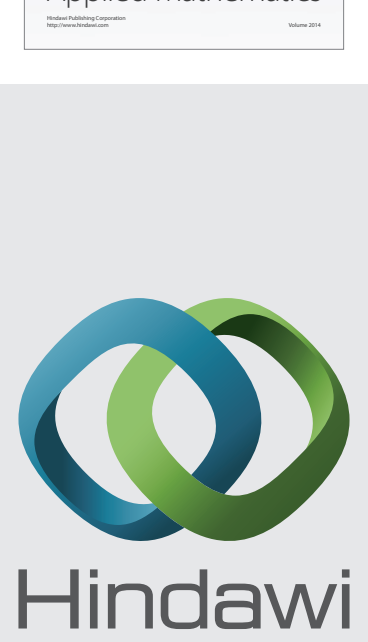

Submit your manuscripts at http://www.hindawi.com
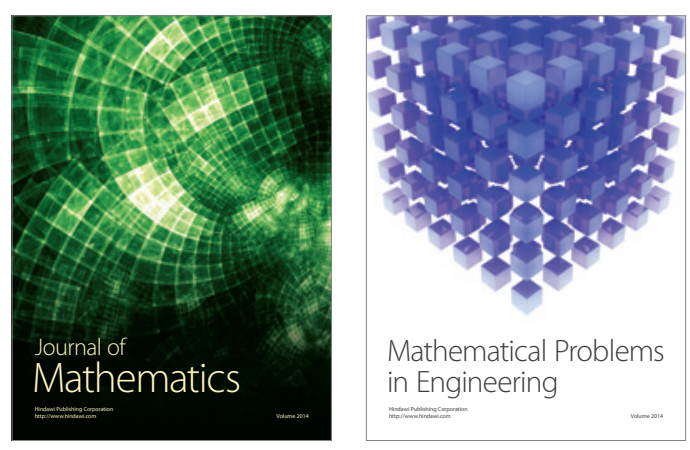

Mathematical Problems in Engineering
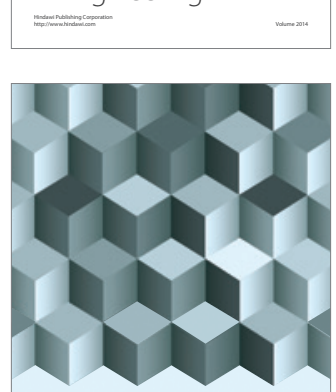

Journal of

Function Spaces
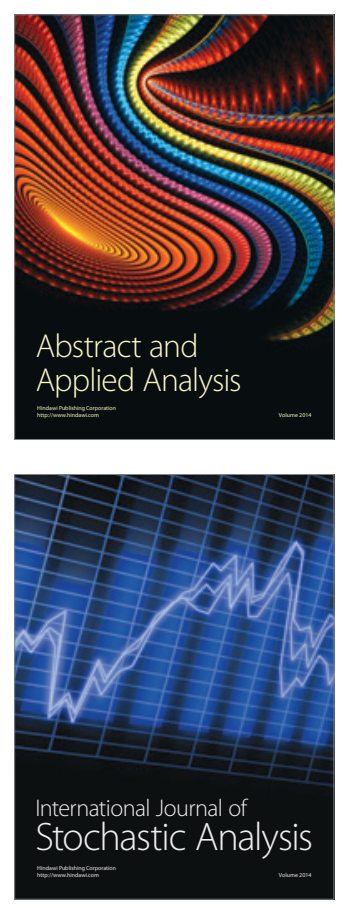

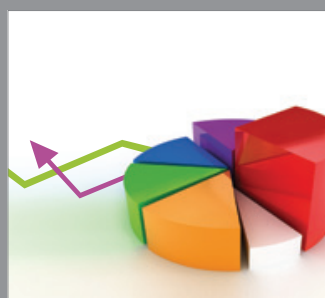

ournal of

Probability and Statistics

Promensencen
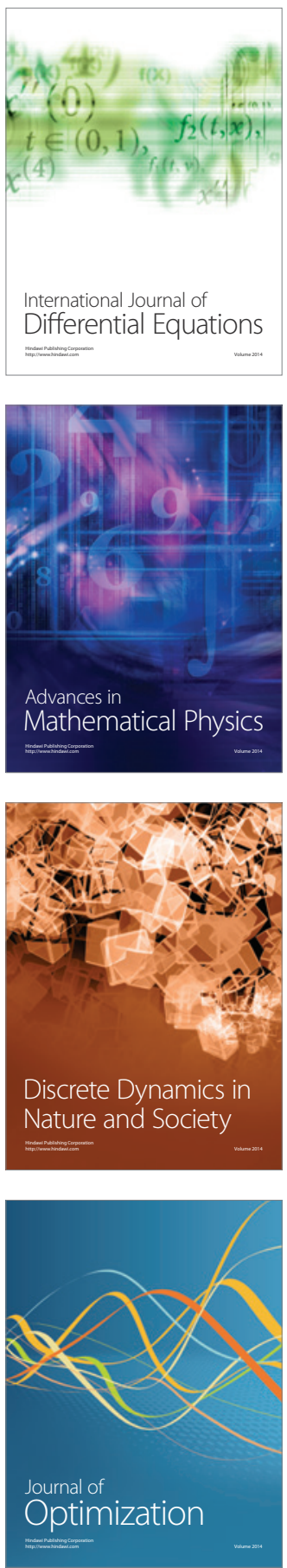